\title{
A Simulation Study on Ultrasound Backscattering by Cell Aggregates with Poly-disperse Cells
}

\author{
Ratan K Saha and Michael C Kolios \\ Department of Physics, Ryerson University \\ 350 Victoria Street, Toronto. M5B 2K3, Canada \\ Emails: ratank.saha@gmail.com, mkolios@ryerson.ca
}

\begin{abstract}
A Monte Carlo simulation study on ultrasound backscattering by cell aggregates with poly-disperse cells is discussed. The nuclei in a cell aggregate were assumed as scatterers and the Anderson model was used to obtain backscattering amplitude for each nucleus. The resultant backscatter echo from many particles was determined by using linear superposition of backscatter signals emitted by the nuclei. The random sequential adsorption (RSA) method was employed to generate spatial organizations of nuclei. The frequency dependent backscattering coefficient (BSC) and signal envelope statistics were obtained from tissue samples with different size distributions. For each poly-disperse sample the nuclear populations followed a Gaussian distribution with the nuclear packing fraction fixed at $50.36 \%$. It was found that integrated backscattering coefficient (IBSC) computed between 10-30 $\mathrm{MHz}$ increased about $7 \mathrm{~dB}$ for the highest poly-disperse sample considered compared to that of a mono-disperse sample. A Gaussian input pulse was employed to investigate signal envelope statistics. It was found that envelope histograms followed the Rayleigh distribution. The Rayleigh fit parameter $(\sigma)$ increased as dispersity increased. For example, for the highest poly-disperse sample, $\sigma$ increased about $105 \%$ and $157 \%$ compared to mono-disperse sample for input pulses with 5 and $25 \mathrm{MHz}$ as the center frequencies and $80 \%$ bandwidths. The present work shows that poly-dispersity contributes to ultrasound backscatter but the shapes of histograms did not vary with the size distribution of scatterers.
\end{abstract}

Keywords: AML Cells, Cell aggregates, Monte Carlo simulation, Ultrasound backscatter, Poly-disperse cells.

\section{INTRODUCTION}

Quantitative ultrasound (QUS) has been extensively used to diagnose and monitor cancer. In this method radio frequency backscatter signals have been analyzed in the spectral domain to quantify scattering properties (e.g. number density of scatterers, average scattering strength, mean scatterer size etc.) of a tissue sample. Such a method becomes useful to localize lesions and to differentiate benign and malignant cancer tissues [1].

Our group has also employed this method to detect cell death in cell samples and tissues exposed to cancer therapies such as chemotherapy, radiation and photodynamic therapy $[2,3]$. It was observed that the backscatter signal intensity increased significantly for apoptotic cells compared to that of viable cells. An approximately 16 fold increase was measured due to apoptosis. Other forms of cell death namely oncosis and mitotic arrest/catastrophe have also been detected and characterized using QUS [3]. These experimental studies revealed that QUS has the potential to provide a noninvasive method to monitor cell death. The differentiation and quantification of various forms cell death are important to assess efficacy of cancer treatments.

Cancer treatments aim to kill abnormal cells and in the process induce various changes at cellular and nuclear morphology. For example, cellular/nuclear spatial organization and size distribution seem to be affected most and that might result in a detectable change in ultrasound backscatter. In this work we examine the role of nuclear size distributions on ultrasound backscattering.

We present a theoretical model, which can account for the size heterogeneity of scatterers and thus provides a realistic simulation tool to study backscattering by cell aggregates with different cellular size distributions. A Monte Carlo algorithm was used to simulate tissue realizations with mono-disperse and poly-disperse nuclei. The backscatter signals generated from those simulations were used to investigate the effects of scatter size distributions on ultrasound backscattering.

\section{MATERIALS AND METHODS}

\section{A. Physical model}

The cells in an aggregate are tightly packed and therefore nuclei can be hypothesized to act as scatterers since their acoustic impedance is different with respect to that of the ambient medium (cytoplasm). Further, the nuclei can be assumed as weak scatterers of incident ultrasound waves because their acoustic properties do not vary much with respect to that of the surrounding medium. Thus, the effects of multiple scattering can be neglected and the resultant backscatter echo signal can be obtained by using linear superposition of scattered signals by the nuclei. The analytical expression of backscattering amplitude for poly-disperse scatterers embedded in a homogeneous loss-less medium for the entire frequency range can be presented as [3]:

$$
\chi_{b}(-2 \mathbf{k})=m\left\langle\frac{1}{N}\left|\sum_{n=1}^{N} \exp \left(i 2 \mathbf{k} \cdot \mathbf{r}_{n}\right) \phi_{b n}\left(k, a_{n}\right)\right|^{2}\right\rangle,
$$

where, $m$ and $\phi_{b n}$ are the number density of scatterers and backscattering amplitude in the Anderson model for the n-th particle with radius $a_{n}$ and position vector $\mathbf{r}_{n}$ respectively. The symbol \langle\rangle represents the ensemble average. This is a general expression of backscattering coefficient and is valid for 


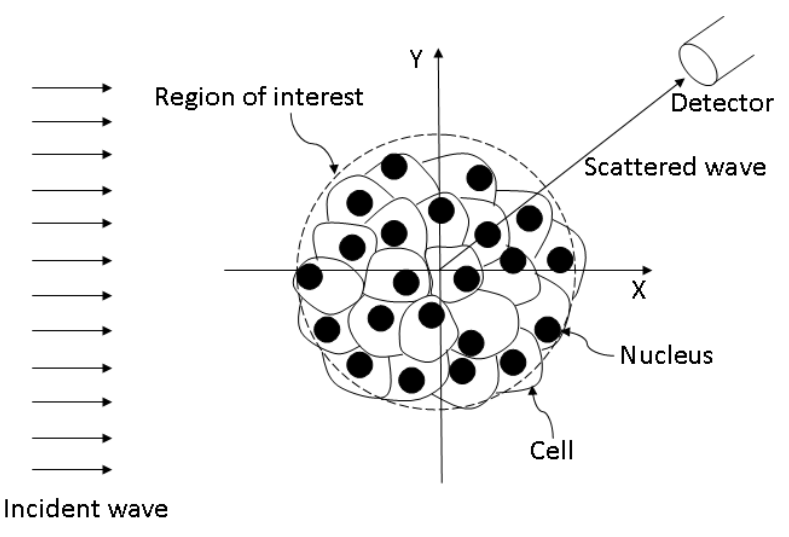

Fig. 1. Scattering diagram (top view).

a poly-disperse medium as well. The corresponding scattering diagram is shown in Fig. 1. For a mono-disperse medium the backscattering amplitude can be factored out from the summation and hence reduces to:

$$
\chi_{b}(-2 \mathbf{k})=m\left\langle\frac{1}{N}\left|\sum_{n=1}^{N} \exp \left(i 2 \mathbf{k} \cdot \mathbf{r}_{n}\right)\right|^{2}\right\rangle\left|\phi_{b}(k, a)\right|^{2} .
$$

An analogous expression has been used extensively to describe ultrasound backscattering by red blood cells [5]. The second term on the right hand side of (2) is referred in the acoustic literature as structure factor of the medium. In the low frequency range for non-overlapping cells it can be approximated by the Percus-Yevick packing factor and in two dimensions (2) can be written as:

$$
\chi_{b}(-2 \mathbf{k})=m W\left|\phi_{b}(k, a)\right|^{2},
$$

where, $W=(1-H)^{3} /(1+H)$, is the Percus-Yevick packing factor in two dimensions and $H$ is the packing fraction of the scatterers. Equations (1) and (2) have been computed in this work to simulate BSCs from poly-disperse and mono-disperse samples. Equation (3) has also been computed to compare its prediction with that of (1) and (2).

The backscatter signal at a large distance $r$ from a collection of particles due to the scattering of a pulse can be expressed as:

$$
\begin{gathered}
p_{s}(r) \approx \frac{1}{\sqrt{2 \pi}} \int d k \frac{e^{i k r}}{r} \mathcal{F}(k)\left[\sum_{n=1}^{N} \exp \left(-\frac{y_{n}^{2}}{2 \sigma_{y}^{2}}\right)\right. \\
\left.\phi_{b n}\left(k, a_{n}\right) e^{i 2 k x_{n}}\right],
\end{gathered}
$$

where, it is assumed that the input pulse is a Gaussian pulse propagating along the $\mathrm{x}$-axis and is given by:

$$
p_{i n}(x, y) \approx\left(\frac{1}{\pi \sigma_{x}^{2}}\right)^{\frac{1}{4}} \exp \left(-\frac{y^{2}}{2 \sigma_{y}^{2}}\right) \exp \left(-\frac{x^{2}}{2 \sigma_{x}^{2}}\right) e^{i k_{0} x},
$$

The notations $\sigma_{y}$ and $\sigma_{x}$ express the beam width and the pulse width respectively. The center frequency of the pulse is defined by $k_{0}$. The Fourier transform (for the $\mathrm{x}$ component only) of the above function is:

$$
\begin{aligned}
\tilde{p}_{i n}(k, y) & =\left(\frac{1}{\pi \sigma_{k}^{2}}\right)^{\frac{1}{4}} \exp \left(-\frac{y^{2}}{2 \sigma_{y}^{2}}\right) \exp \left(-\frac{\left(k-k_{0}\right)^{2}}{2 \sigma_{k}^{2}}\right) \\
& =\exp \left(-\frac{y^{2}}{2 \sigma_{y}^{2}}\right) \mathcal{F}(k)
\end{aligned}
$$

and $\sigma_{x}=1 / \sigma_{k}$. It should be noted in (4) that the individual backscattering amplitude for each particle is weighted by the strength of the pressure field at that location in order to obtain the resultant backscatter signal from many particles. Equation (4) has been computed in this study to investigate signal envelop statistics from different samples with various size distributions of nuclei.

\section{B. Simulation method}

The size of the region of interest (ROI) was taken as $1000 \mathrm{x}$ $1000 \mu \mathrm{m}^{2}$ for BSC computation and this region was assumed to be completely filled by the acute myeloid leukemia (AML) cells. Thus nuclei could be assumed to be the scatterers. The packing fraction was fixed to $H=50.36 \%$ for a sample with uniform nuclear size. This value is close to the nuclear to cell volume ratio $(\approx 0.55)$ of that cell line and also close to the maximum area packing fraction $(52-55 \%$ [6]) in two dimensions by discs with equal radii. For poly-disperse samples nuclear populations, when plotted as a function of their radius, followed Gaussian distributions but the total area occupied by the nuclei always remained constant to $50.36 \%$. The density and speed of sound within the nucleus were chosen as $\rho_{e}=1180 \mathrm{~kg} / \mathrm{m}^{3}$ and $c_{e}=1523 \mathrm{~m} / \mathrm{s}$ respectively. The numerical values of the same quantities of the surrounding medium were taken as $\rho_{0}=1000 \mathrm{~kg} / \mathrm{m}^{3}$ and $c_{0}=1483 \mathrm{~m} / \mathrm{s}$ respectively [7].

A Monte Carlo algorithm known as random sequential adsorption (RSA) method was used to assign the coordinates of the particles. In this algorithm a coordinate of a particle was proposed and was accepted if it did not overlap with the existing particles under periodic boundary conditions. Otherwise this location was canceled and a new position was assigned. Thus the scatterers might touch but would not overlap. For a poly-disperse sample we implemented the same algorithm to generate tissue realizations but we placed the largest nuclei at first and then gradually nuclei with lower sizes were positioned within the region of interest [8].

To study signal envelope statistics we approximated the incident ultrasound beam emitted by a circular transducer as a Gaussian beam. Scattering of two separate input pulses with 5 and $25 \mathrm{MHz}$ as the center frequencies were examined to study bandwidth dependent behaviors. A $80 \%$ bandwidth was fixed for each pulse. Thus, the pulse widths became $\sigma_{x}=0.137$ $\mathrm{mm}$ and $0.027 \mathrm{~mm}$ respectively. Corresponding values of beam width were taken as $\sigma_{y}=0.89 \mathrm{~mm}$ and $0.45 \mathrm{~mm}$ respectively for these pulses. In this case a $4000 \times 4000 \mu \mathrm{m}^{2}$ ROI was considered to generate sufficiently long A-lines. 

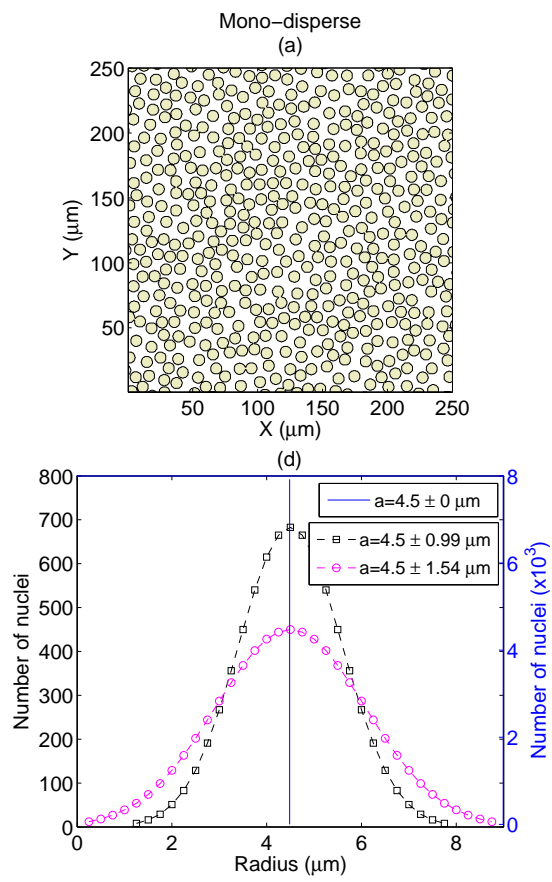

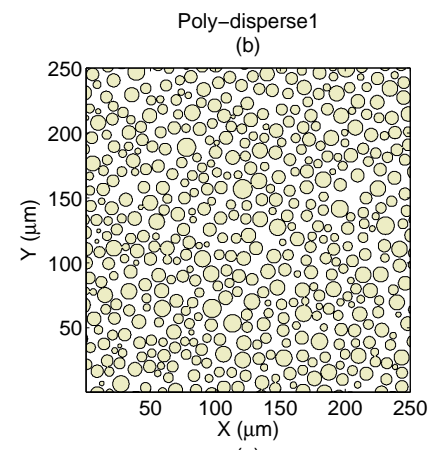

(e)

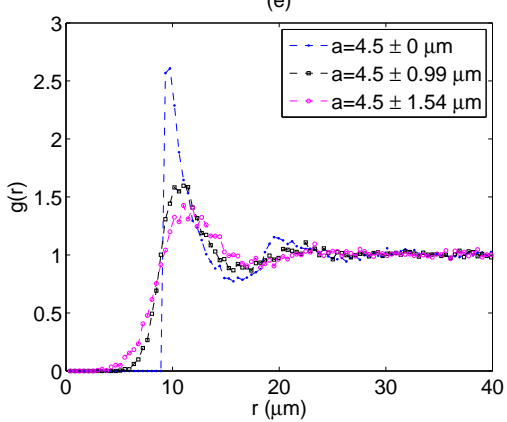

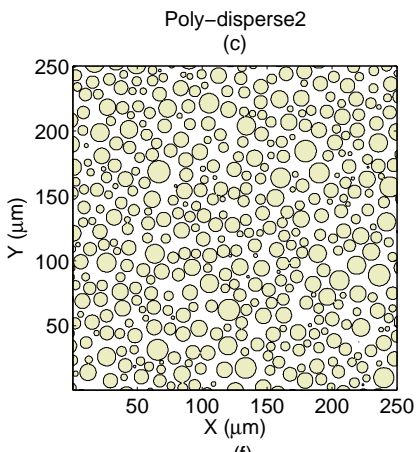

$(\mathrm{f})$

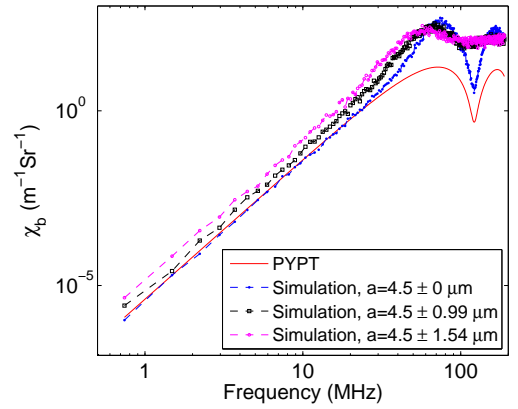

Fig. 2. Simulated tissue realizations with mono-disperse [(a)] and poly-disperse [(b) and (c)] nuclei. (d) Plot of number of nuclei as a function of radius. The mean scatterer size and standard deviation are given in the legend for each sample. (e) Variation of the pair correlation function with distance for each sample. (f) Frequency dependent backscattering coefficient for each sample along with Percus-Yevick prediction (PYPT).

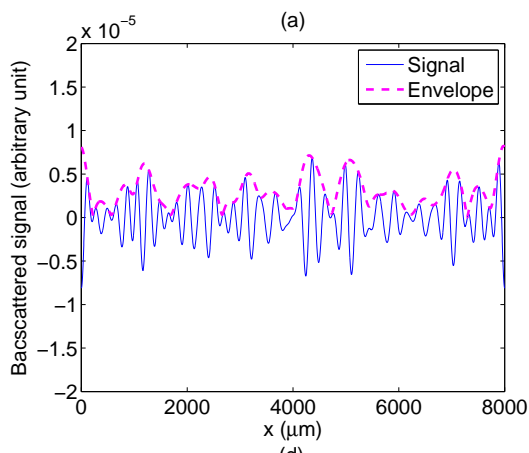

(d)

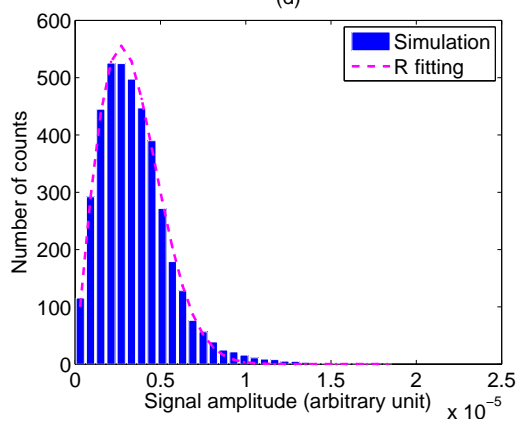

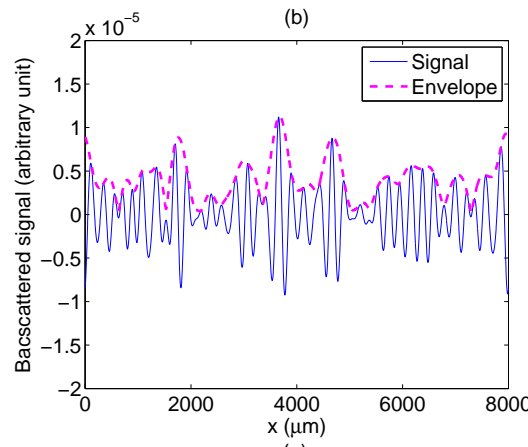

(e)

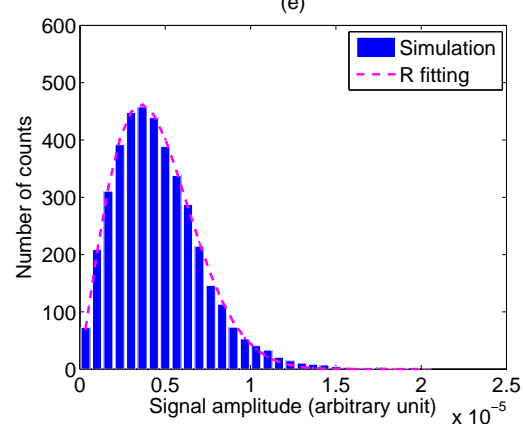

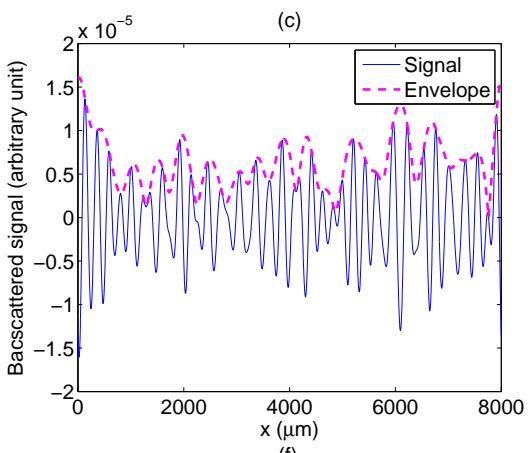

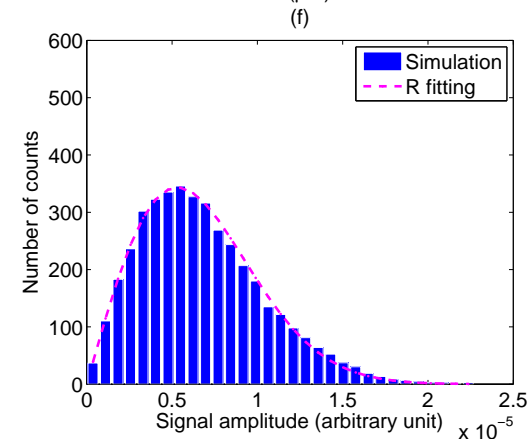

Fig. 3. (a)-(c) Simulated [by using (4)] backscatter A-lines from those samples (Mono-disperse, Poly-disperse1 and Poly-disperse2) respectively for the scattering of a $5 \mathrm{MHz}$ Gaussian input pulse. (d)-(f) Corresponding histograms for 100 A-lines and the Rayleigh fitted curves. 


\section{RESULTS}

A two dimensional packing of nuclei with uniform size mimicking a cell aggregate realization is shown in Fig. 2(a). Figs. 2(b) and (c) represent configurations of aggregates with poly-disperse nuclei. For clarity smaller region of interest (250 × $250 \mu \mathrm{m}^{2}$ ) was shown for each sample. Corresponding nuclear populations are presented as a function of their size in Fig. 2(d). For mono-disperse sample it follows a uniform distribution (right axis) whereas for poly-disperse samples they followed Gaussian distributions. The variation of pair correlation function for each sample is plotted in Fig. 2(e) to display its structural properties. This figure shows that for the first sample $g(r)$ is zero for $r<9 \mu \mathrm{m}$ and this is due to fact that rigid nuclei cannot overlap. However, for other cases this restriction is no longer valid because of the presence of smaller particles in the samples. Moreover, the samples have similar structural properties at a distance $>25 \mu \mathrm{m}$. The frequency dependent backscattering coefficient curves are displayed in Fig. 2(f). It is evident that BSC increased up to $50 \mathrm{MHz}$ as poly-dispersity increased. For example, IBSC for the third sample computed between $10-30 \mathrm{MHz}$ is about $7 \mathrm{~dB}$ more than that of the first sample as given in Table I. However, associated SS did not vary significantly (see Table I). In the higher frequency range $(>50 \mathrm{MHz})$ instead of oscillatory behavior (which is evident in the mono-disperse case) smother variations of BSC curves are observed for poly-disperse samples. For the later samples the maxima and minima positions are different for different nuclear sizes and thus smother curves were obtained.

TABLE I

VARIATIONS OF IBSC, SS AND RAYLEIGH FIT PARAMETER $(\sigma)$ WITH POLY-DISPERSITY.

\begin{tabular}{|c|c|c|c|c|}
\hline Sample & IBSC (dB) & SS & $\begin{array}{c}\sigma \text { (a.u.) } \\
\text { for 5 MHz }\end{array}$ & $\begin{array}{c}\sigma \text { (a.u.) } \\
\text { for } 25 \mathrm{MHz}\end{array}$ \\
\hline Mono-disperse & - & 4.17 & $2.59 \mathrm{e}-9$ & $4.98 \mathrm{e}-5$ \\
Poly-disperse1 & 3.29 & 4.26 & $3.59 \mathrm{e}-6$ & $8.41 \mathrm{e}-5$ \\
Poly-disperse2 & 6.99 & 4.47 & $5.31 \mathrm{e}-6$ & $12.81 \mathrm{e}-5$ \\
\hline
\end{tabular}

Fig. 3(a) illustrates a backscatter A-line from the first sample for the scattering of $5 \mathrm{MHz}$ pulse. The envelope of that A-line is also outlined in the same figure. Figs. (b) and (c) display A-lines form other samples. Corresponding histograms are plotted in the second row. It is clear from the figures and from the table that as the dispersity increased signal strength also increased. For instance, for the highest poly-disperse sample $\sigma$ increased about $105 \%$ compared to that of monodisperse sample for $5 \mathrm{MHz}$ pulse (see Table I). The backscatter signals for $25 \mathrm{MHz}$ pulse are not been shown but numerical values are presented in Table I. In this case similar signal characteristics can be observed. Therefore, size heterogeneity of scatterers did not affect shapes of envelope histograms.

\section{Conclusion}

A Monte Carlo study on ultrasound backscattering by AML cells in aggregates was discussed here. In this study, the backscatter signals were obtained from aggregates with mono-disperse and poly-disperse nuclei. The first prototype mimicked spatial distributions of nuclei of viable cells in aggregates. The other prototypes imitated nuclear organizations of apoptotic cells in pellets, which would have a greater distributions of nuclear size. Thus it was possible to the investigate role of size distributions of particles on ultrasound backscattering. A simulation algorithm referred as random sequential adsorption technique was implemented to generate some configurations of those samples. Both frequency dependent backscattering coefficient and signal envelope statistics were examined.

The theoretical model presented here can accommodate heterogeneous sizes of scatterers and thus provides a realistic frame work to simulate ultrasound backscattering by samples with poly-disperse nuclei. It was found that IBSC increased about $7 \mathrm{~dB}$ for the highest poly-disperse sample compared to that of mono-disperse sample and associated spectral slope also increased but marginally. Corresponding Rayleigh fit parameter increased nearly $105 \%$ and $157 \%$ for the scattering of 5 and $25 \mathrm{MHz}$ Gaussian input pulses respectively. Thus this model is capable to generate physically meaningful results and also consistent with experimental findings [3]. In future it would be interesting to extend this study for three dimensional distributions of cells.

\section{ACKNOWLEDGMENT}

This work was supported by the Canadian Institutes of Health Research grant MOP-97959 and was undertaken, in part, thanks to funding from the Canada Research Chairs Program awarded to M. C. K. This work was made possible by the facilities of the Shared Hierarchical Academic Research Computing Network (SHARCNET:www.sharcnet.ca) and Compute/Calcul Canada. We would like to thank the "Rseau Qubcois de Calcul de Haute performance" (RQCHP) for generous allocation of computer resources. We also like to thank Prof. J Hunt and Mr. R Baddour for many helpful discussions and comments.

\section{REFERENCES}

[1] M. L. Oelze and J. F. Zachary, Ultrasound Med. Biol., 32(11), 1639, 2006.

[2] G. J. Czarnota et al., Ultrasound Med. Biol., 23(6), 961, 1997.

[3] R. M. Vlad et al., Ultrasound Med. Biol., 36(9), 1546, 2010.

[4] P. M. Morse and K. U. Ingard, "Theoretical Acoustics", McGraw-Hill Book Company, 1968.

[5] D. Savéry and G. Cloutier, IEEE Trans. Ultrason. Ferroelectr. Freq. Control, 52(1), 94, 2005.

[6] E. L. Hinrichsen, J. Feder, T. Jøssang, Phys. Rev. A, 41, 4199, 1990.

[7] R. E. Baddour and M. C. Kolios, J. Acoust. Soc. Am., 121(1), EL16, 2006.

[8] T. Aste, Phys. Rev. E, 53(3), 2571, 1996. 